\title{
Urinary Calculi: A Microbiological and Biochemical Analysis at a Tertiary Care Hospital in Eastern Nepal
}

\author{
Pratima Shah ${ }^{1},{ }^{1}$ Ratna Baral, ${ }^{1}$ C. S. Agrawal, ${ }^{2}$ Madhab Lamsal, ${ }^{3}$ Dharanidhar Baral, ${ }^{4}$ \\ and Basudha Khanal ${ }^{1}$ \\ ${ }^{1}$ Department of Microbiology \& Infectious Diseases, B.P. Koirala Institute of Health Sciences (BPKIHS), Dharan, \\ Sunsari 56700, Nepal \\ ${ }^{2}$ Department of Surgery, B.P. Koirala Institute of Health Sciences (BPKIHS), Dharan, Sunsari 56700, Nepal \\ ${ }^{3}$ Department of Biochemistry, B.P. Koirala Institute of Health Sciences (BPKIHS), Dharan, Sunsari 56700, Nepal \\ ${ }^{4}$ School of Public health and Community Medicine, B.P. Koirala Institute of Health Sciences (BPKIHS), Dharan, \\ Sunsari 56700, Nepal
}

Correspondence should be addressed to Pratima Shah; pratimashahdr@yahoo.com

Received 29 June 2020; Revised 19 August 2020; Accepted 2 September 2020; Published 12 September 2020

Academic Editor: Luigi Santacroce

Copyright (C) 2020 Pratima Shah et al. This is an open access article distributed under the Creative Commons Attribution License, which permits unrestricted use, distribution, and reproduction in any medium, provided the original work is properly cited.

Background. The occurrence of urinary tract infection in presence of urolithiasis is frequently noted; however, microbial agents of urolithiasis and their antimicrobial susceptibility patterns remain underinvestigated. This study aimed to identify the microorganisms isolated from urine and stone matrices to determine their antimicrobial susceptibility, to find the association between the pathogens of urine and stone matrices, and to perform the biochemical analysis of stones. Methods. A total of 88 cases of urolithiasis admitted for elective stone removal at Department of surgery, B.P. Koirala Institute of Health Sciences (BPKIHS), were enrolled. Preoperative urine culture and postoperative stone culture were performed. Isolation, identification, and AST were done by the standard microbiological technique. Further qualitative biochemical analysis of stones was also attempted. Result. Among 88 stone formers recruited, culture of urine, whole stone, and nidus yielded the growth of bacteria 44, 32, and 30, respectively. Bacteria isolated from urine culture correlated with those from stone matrices with a sensitivity of $90 \%$, specificity of $79.69 \%$, PPV of $63.64 \%$, and NPV of $95.45 \%$. Escherichia coli (46.7\%) was the most common bacteria followed by Klebsiella pneumoniae (16.7\%) and Proteus mirabilis (13.3\%) from urine and stone cultures. Almost all the uropathogens isolated were susceptible to commonly used antibiotics. Calcium oxalate $(84.1 \%)$ was common biochemical constituent found in stone formers followed by calcium oxalate + phosphate $(8 \%)$. Conclusions. The association of microorganism isolated from urine and nidus culture was significant that can predict the source of infective stone; however, in some cases, microorganisms and the antimicrobial susceptibility pattern from urine and nidus were different. This study emphasizes the use of appropriate antimicrobial agents to prevent the regrowth of residual stones and minimize the risk of infectious complications after surgical removal of stones.

\section{Introduction}

Urolithiasis is one of the frequently encountered urological disorders, common throughout the world, and is highly prevalent in Nepal [1].

The association between urolithiasis and urinary tract infections (UTIs) is well known and is frequently detected. Their interrelationship can be defined in two ways: urolithiasis following UTIs, i.e., "infection-induced stones" or urinary stone with subsequent UTIs as its complications [2]. Although the calculi themselves are the important source of secondary infection, their prevalence, causative microorganisms, and antimicrobial susceptibility patterns in Nepal remain underinvestigated. Approximately, $15 \%$ of urinary stones are infective stones. However, formation of all noninfective urinary calculi is a consequence of unknown changes in kidney tissue or metabolic disturbances [3].

The history describes infective stone or struvite as the most common type of urinary stones containing magnesium 
ammonium phosphate, whereas urea-splitting bacteria like Proteus spp., Staphylococcus aureus, Klebsiella spp., Providencia spp., and Ureaplasma urealyticum are commonly responsible for struvite stone. The antimicrobial agents could not invade, where these bacteria lie within the interspace of stones in urinary tract. Thus, the outcome is progressive expansion of stones because of persistent infection over a period of weeks or months $[2,4]$.

To prevent infectious complications and subsequent recurrence of residual stones after surgical removal, association of microbes in the stone and proper antibiotic therapy are essential. The selection of antibiotic agents is based on bacteria isolated from urine culture; however, the efficacy of treatment of stone bacteria cannot be ascertained due to uncertainty in similarity of stone and urine bacteria.

Similarly, biochemical profile of the patient should be evaluated to ameliorate metabolic disorder and to inhibit reoccurrence of metabolic stone. Therefore, characterization of calculus material aids knowledge to establish the management of a patient postoperatively $[5,6]$.

Thus, this study was aimed to identify the type of bacteriology of urine and stone matrices, determine their antimicrobial sensitivity pattern, and find the concordance between urine and stone bacteria. It was also aimed to determine the biochemical composition of urinary calculi to know the origin and etiology of urinary stones.

\section{Methods}

This cross-sectional descriptive study was conducted in the Department of Microbiology in collaboration with Department of General Surgery and Biochemistry. A total of 88 patients having urolithiasis, admitted at B. P. Koirala Institute of Health sciences, Dharan, Nepal, for elective stone removal between July 2014 and June 2015 were enrolled. Informed consents were taken from the patients, and ethical clearance was obtained from the Institutional Review Committee of BPKIHS (Code No: IERB/277/014).

The demographic details and associated factors with urolithiasis such as past and family history of stone disease, history of hypertension, diabetes mellitus, and gout were recorded in predesigned proforma.

\subsection{Sample Preparation, Isolation, and Identification of} Bacteria [7-9]. The midstream urine specimen was cultured from each patient before surgical stone removal. The stone was also collected from the same patient after surgery.

Using the semiquantitative method, $10^{5}$ colony-forming units per milliliter $(\mathrm{CFU} / \mathrm{ml})$ of urine was considered as significant bacteriuria and further processed for identification of organisms. The bacterial pathogens were identified up to species level by standard microbiological techniques like colony morphology, Gram staining, and several biochemical tests.

Antimicrobial susceptibility of the isolates was determined by the Kirby-Bauer disc-diffusion method on Muller-Hinton agar (MHA) according to Clinical Laboratory Standards Institute (CLSI) guidelines [10].
2.2. Preparation and Processing of Urinary Stone [2]. After the surgical removal, the stone sample was washed several times with sterile water and each stone was then divided into two parts, as symmetrical as possible. For the first part, stone matrices were taken from "nidus" (nucleus) portion by scraping. For the second part, the stone was crushed into powder by sterilized mortar and pestle and was then used for bacterial culture and chemical analysis of the "whole stone". All samples derived from two locales of individual stones (including nidus and whole stone) were then inoculated in brain-heart infusion (BHI) broth, incubated at $35^{\circ} \mathrm{C}$ for $6-8$ hours, inoculated onto blood, MacConkey, CLED agar (HiMedia Laboratories), and incubated at $35^{\circ} \mathrm{C}$ for 24 and 48 hours, respectively.

Bacteria isolated thus were identified by standard biochemical tests. Antimicrobial susceptibility was determined by the disc diffusion assay on MHA (HiMedia Laboratories) following the standards of CLSI [10].

2.3. Analysis of Chemical Compositions of Stones. The qualitative biochemical analysis of compositions of each stone was done for carbonate, calcium, magnesium, phosphate, oxalate, uric acid, and cysteine, using stone powder derived from the second part of stone sections (as aforementioned) that was left after bacterial culture [11].

2.4. Statistical Analysis. All data collected were entered in MS excel 2007 and analysed using SPSS 21.0. For descriptive analysis, percentage and ratio were calculated with tabular and graphical presentation of analysis.

For inferential statistics, the chi-square test was applied to find out the relationship between dependent and independent variables. $P$ values $<0.05$ were considered statistically significant.

\section{Results}

Out of eighty-eight patients, 50 were males with an average age of 38.3 (range: 17-67) years and 38 were females with an average age of 41.2 (range: 18-69) years. The male: female ratio was $1.3: 1$. Forty had nephrolithiasis (45.5\%), thirtyseven had ureterolithiasis (42\%), and eleven had urinary bladder stone (12.5\%).

The comorbidities were observed in 23 (27.1\%) patients. Hypertension was present in $18(78.3 \%)$ patients, and 6 (26.1\%) had diabetes mellitus. Only $1(4.35 \%)$ of the patients was found to have gout, and a past history of urolithiasis was present in $2(2.272 \%)$.

3.1. Urine and Stone Matrices Culture. Bacterial growth was obtained in urine of 44 patients (50\%). The most common organism isolated was Escherichia coli (20 (45.5\% of total isolates)) followed by Klebsiella pneumoniae (7 (15.9\%)), Proteus mirabilis (4 (9.1\%)), Enterococcus faecalis (4 (9.1\%)), and Staphylococcus aureus (3 (6.8\%)). 
When attempted stone culture, whole stone of 32 (36.4\%) out of 88 patients yielded the growth of bacteria, whereas nidus culture bacteria in 30 (34.1\%). The most common organism isolated from whole stone and nidus culture was Escherichia coli followed by Klebsiella pneumoniae, Proteus mirabilis, Enterococcus faecalis, and Staphylococcus aureus. Infective stone was found in $34.1 \%$ of the cases. The culture results and bacterial isolates are shown in Table 1.

3.2. Association of Bacteria Isolated from Urine and Stone Matrices Culture. Bacteria isolated from urine were similar to those obtained from stone matrices. Considering culture of stone matrices a gold standard, sensitivity and specificity of urine culture were found to be $93.3 \%$ and $72.4 \%$, respectively. The PPV and NPV were $63.6 \%$ and $95.5 \%$, respectively (Table 2).

\subsection{Similarity of Bacteria Isolated from Urine and Stone Matrices Culture}

3.3.1. Concordance between Microorganisms Isolated from Urine and Stones Matrices Culture. Some bacteria isolated from urine were phenotypically similar to those from stone matrices. Out of 30 stone formers, a total of 30 bacteria were isolated. Among those 30 stone formers, 28 had bacterial isolates in both urine and stone matrices, whereas no organism was obtained from urine in only 2 of the patients. Among 28 stone formers, 24 had the same organism and 3 had different strains of $E$. coli found in urine and stone matrices, whereas the remaining 1 had different isolates, i.e., E. coli from urine and K. oxytoca from stone matrices. The bacteria isolated from urine were common to stone matrices in context of whole stone culture also. The culture results are shown in Tables 3 and 4.

\subsection{Antimicrobial Susceptibility Pattern (AST) of Urinary Pathogen}

3.4.1. Antimicrobial Susceptibility Pattern of Gram-Negative Bacteria. All Gram-negative bacteria isolated were sensitive to imipenem (100\%) followed by gentamicin (94\%), amikacin $(91 \%)$, nitrofurantoin $(88 \%)$, ceftazidime $(87.9 \%)$, and cotrimoxazole $(58 \%)$, and most of them were resistant to ampicillin (91.6\%) and ofloxacin (69\%). Out of 20 E. coli, seven (35\%) E. coli were ESBL producers (Figure 1).

3.5. Antimicrobial Susceptibility Pattern of Gram-Positive Bacteria. All Gram-positive bacteria, S. aureus, E. faecalis, and CONS isolated, were sensitive to vancomycin (100\%), linezolid $(100 \%)$, gentamicin $(100 \%)$, and nitrofurantoin $(100 \%)$ followed by amikacin (87.5\%), norfloxacin $(87.5 \%)$, ciprofloxacin $(75 \%)$, cotrimoxazole $(62.5 \%)$, and ofloxacin (50\%). Out of 3 isolates of $S$. aureus, 2 were MRSA.

\subsection{Antimicrobial Susceptibility Pattern of Bacteria Isolated from Stone Matrices (Nidus)}

3.6.1. Antimicrobial Susceptibility Pattern of Gram-Negative Bacteria. All Gram-negative bacteria isolated were sensitive to imipenem (100\%) followed by gentamicin (96\%), amikacin $(84.6 \%)$, nitrofurantoin $(88.5 \%)$, ceftazidime $(65.4 \%)$, norfloxacin $(65.4 \%)$, ciprofloxacin $(53.8 \%)$, cotrimoxazole (34.6\%), and ofloxacin (34.6\%) and were resistant to ampicillin (92\%). Out of total isolates, 9 were ESBL producers (Figure 2).

3.7. Antimicrobial Susceptibility Pattern of Gram-Positive Bacteria. All Gram-positive bacteria were sensitive to vancomycin (100\%), linezolid (100\%), gentamicin $(100 \%)$, and nitrofurantoin (100\%) followed by ceftriaxone $(50 \%)$, cotrimoxazole (50\%), amikacin (50\%), and ciprofloxacin (50\%), and most of them were resistant to ofloxacin (100\%). Out of 3 isolates of $S$. aureus, 2 were MRSA.

3.8. Stone Analysis. The qualitative biochemical stone analysis was attempted in all the stones, and the most common type was found to be calcium oxalate $(74(84.1 \%))$ followed by calcium oxalate phosphate $(7(8.0 \%))$, ammonia (3 $(3.4 \%))$, calcium uric acid $(2(2.3 \%))$, and calcium carbonate $(1(1.1 \%))$.

3.9. Bacteria Associated with Stone. Among 74 calcium oxalate stones, 23 (71.9\%) had association with stone matrices (nidus) bacteria. Most common stone formers were $E$. coli followed by K. pneumoniae. Proteus mirabilis was associated with ammonia and calcium oxalate with magnesium and phosphate. Bacterial isolates from nidus culture were associated with almost all types of urinary stones (Figure 3).

\section{Discussion}

This study made an attempt to identify the type of bacteriology of urine and stone matrices, to determine their antimicrobial sensitivity pattern and to find the concordance between urine and stone bacteria in patients with urolithiasis.

4.1. Demographic Factors. The age of the patients in our study ranged from 17 years to 69 years, frequency being highest in age groups of 30-39 years (29.5\%), a finding contradictory to previous studies conducted which documented that the frequency of urinary stones increased with age.

Among the study participants, 50 (56.8\%) were male and 38 (43.2\%) were female. Although nephrolithiasis continues to be more common in men, the male-to-female ratio with urinary tract stones has narrowed from 3.1 to 1.3 from 1970 to 2000 [12] and from $1.6: 1$ to $1.2: 1$ from 1998 to 2003 [13]. The striking new trends of increased incidence of stone formation in women might be due to associated risk factors 
TABLE 1: Pattern of bacteria isolated from urine and stone matrices culture.

\begin{tabular}{lccc}
\hline Bacteria & $\begin{array}{c}\text { Urine } \\
\text { No. of patients (\%) }\end{array}$ & $\begin{array}{c}\text { Whole stone } \\
\text { No. of patients (\%) }\end{array}$ & $\begin{array}{c}\text { Nidus } \\
\text { No. of patients (\%) }\end{array}$ \\
\hline Escherichia coli & $20(45.5)$ & $14(43.8)$ & $14(46.7)$ \\
Enterococcus faecalis & $4(9.1)$ & $1(3.1)$ & $1(3.3)$ \\
Klebsiella pneumoniae & $7(15.9)$ & $5(15.6)$ & $5(16.7)$ \\
Proteus mirabilis & $4(9.1)$ & $4(12.5)$ & $4(13.3)$ \\
Staphylococcus aureus & $3(6.8)$ & $4(12.5)$ & $3(10.0)$ \\
Citrobacter koseri & $2(4.5)$ & 0 & 0 \\
Pseudomonas aeruginosa & $1(2.3)$ & $1(3.1)$ & $1(3.3)$ \\
CONS & $1(2.3)$ & 0 & 0 \\
Klebsiella oxytoca & $2(4.5)$ & $2(6.3)$ & $1(3.3)$ \\
Enterobacter spp. & 0 & $1(3.1)$ & $1(3.3)$ \\
Total & $\mathbf{4 4 ( \mathbf { 1 0 0 }}$ & $\mathbf{3 2 ( 1 0 0 )}$ & $\mathbf{3 0 ( \mathbf { 1 0 0 } )}$ \\
\hline
\end{tabular}

TABLE 2: Association of bacteria isolated from urine and stone matrices culture.

\begin{tabular}{|c|c|c|c|c|c|}
\hline \multirow{2}{*}{ Test } & \multirow{2}{*}{$\begin{array}{c}\text { Test } \\
\text { status }\end{array}$} & \multicolumn{2}{|c|}{ Urine culture } & \multirow{2}{*}{$P$ value } & \multirow{2}{*}{ Remarks } \\
\hline & & Positive & Negative & & \\
\hline Nidus & Positive & 28 & 2 & & \\
\hline culture & Negative & 16 & 42 & $<0.001$ & Significant \\
\hline Total & 44 & 44 & & & \\
\hline
\end{tabular}

such as increasing obesity, dietary changes, and change in fluid intake patterns. In the context of our study, male preponderance was observed. It can be attributed to effect of sex hormones on some lithogenic risk factors and concentration of lithogenic factors in the urine which is greater in men than that in women [14-17].

By occupation, 32 (36.4\%) were housewives, 21 (23.9\%) were involved in business, 13 (14.8\%) in service, 9 (10.2\%) in farming, $7(8.0 \%)$ were students, and $6(6.8 \%)$ were laborers. As reported by Vhlensieck et al. [18] and Kadir et al. [19], our data also showed that the frequent occupational group was prone to develop urinary stones with sedentary life styles like housewives, business (shopkeeper), and service holders. Sedentary lifestyle predisposes to sedimentation of urine, and crystals may be trapped by gravity in upward draining collecting tubules or in the inferior calices of the kidney [20].

Regarding comorbid conditions, 18 (20.5\%) respondents were hypertensives and $6(6.8 \%)$ were diabetics. Hypertension with diabetes was seen in 2 cases, while 1 had gout (1.1\%). Several studies have established hypertension as an independent risk factor of urolithiasis [21-23] with a proposal that abnormalities in renal calcium metabolism exist among patients with hypertension, leading to increased urinary calcium excretion [24]. Our findings of 12 hypertensive patients having calcium oxalate stones and one with calcium uric acid stone are in concordance with the abovementioned studies.

Urinary stone disease has been increased globally and occurred more frequently in subjects with diabetes than nondiabetics with a predominance of urolithiasis with uric acid [25-27]. The reason for a higher occurrence of urolithiasis in diabetes mellitus has been explained as insulin resistance and lower urine $\mathrm{pH}$ through impaired kidney ammonia genesis, promoting uric acid stone formation [28].
Although a low urinary $\mathrm{pH}$ plays a major role in the formation of uric acid kidney stones, a defect in renal acid excretion also could lead to hypocitraturia, an important risk factor for calcium stones [29]. In our study, the patient with diabetes had infective stones. The chemical composition of stones varied in different patients such as calcium oxalate, ammonia, and calcium oxalate phosphate, which showed different results than the study done by Medyen et al. and Chu et al. [25, 27].

In the study by Alvarez-Nemegyi et al., 39\% of patients with primary gout had urinary stones, of which about $30 \%$ were silent and diagnosed only by ultrasonography, meaning the prevalence of urolithiasis in gout is likely to be higher than commonly reported [30]. We could not comment upon the association of gout with urolithiasis in our study as we had only one such case. Perhaps, most of the cases with urolithiasis in gout remain silent as stated by AlvarezNemegyi et al. and thus are underreported.

Regarding stone locations, in the present study, 40 (45.5\%) had nephrolithiasis, 37 (42.0\%) ureterolithiasis, and $15(12.5 \%)$ had urinary bladder stones. Upper urinary tract stone constituted $87.5 \%$ and lower urinary tract stone $12.5 \%$ in the ratio of $7: 1$. Our results have been consistent with study of Ahmed et al. [31] who reported increased frequency of renal stones. Kidney acts as a first barrier filter for crystals, thereby damaging tubular epithelium, which acts as a nidus for the stone formation. However, our result was different from the study of Venkatramana who observed the increased frequency of ureteric stone [32]. This variation might be due to selection of the patients irrespective of site of the stones.

Past history of urolithiasis was present in $2(2.3 \%)$ cases. The reason for such low incidence of recurrence in our study may be because of the relatively shorter duration (1 year) of study, whereas many previous studies had followed up the patients for 10 years.

4.2. Urinary Tract Infection and Infective Stone in Urolithiasis. In this study, bacterial growth was obtained in urine of $50 \%$ of patients. Organisms isolated in decreasing order of frequency were Escherichia coli (20 (45.5\%)), Klebsiella pneumoniae (7 (15.9\%)), Proteus mirabilis (4 (9.1\%)), Enterococcus faecalis (4 (9.1\%)), and Staphylococcus aureus (3 $(6.8 \%))$. 
TABLE 3: Similarity in urine and stone matrices (nidus) culture.

\begin{tabular}{|c|c|c|c|c|c|c|c|c|}
\hline \multirow{2}{*}{ Nidus bacteria } & \multicolumn{7}{|c|}{ Urine bacteria } & \multirow{2}{*}{ Tota } \\
\hline & E. coli & E. faecalis & K. pneumoniae & P. mirabilis & S. aureus & P. aeruginosa & K. oxytoca & \\
\hline E. coli & 14 & 0 & 0 & 0 & 0 & 0 & 0 & 14 \\
\hline E. faecalis & 0 & 1 & 0 & 0 & 0 & 0 & 0 & 1 \\
\hline K. pneumoniae & 0 & 0 & 5 & 0 & 0 & 0 & 0 & 5 \\
\hline Proteus mirabilis & 0 & 0 & 0 & 4 & 0 & 0 & 0 & 4 \\
\hline S. aureus & 0 & 0 & 0 & 0 & 2 & 0 & 0 & 2 \\
\hline P. aeruginosa & 0 & 0 & 0 & 0 & 0 & 1 & & 1 \\
\hline K. oxytoca & 1 & 0 & 0 & 0 & 0 & 0 & 0 & 1 \\
\hline Total & 15 & 1 & 5 & 4 & 2 & 1 & 1 & 28 \\
\hline
\end{tabular}

TABLE 4: Similarity in urine and stone matrices (whole) culture.

\begin{tabular}{|c|c|c|c|c|c|c|c|c|}
\hline \multirow{2}{*}{ Whole stone bacteria } & \multicolumn{7}{|c|}{ Urine bacteria } & \multirow{2}{*}{ Tota } \\
\hline & E. coli & E. faecalis & K. pneumoniae & P. mirabilis & S. aureus & P. aeruginosa & K. oxytoca & \\
\hline $\begin{array}{l}\text { E. Coli } \\
\end{array}$ & 14 & 0 & 0 & 0 & 0 & 0 & 0 & 14 \\
\hline E. faecalis & 0 & 1 & 0 & 0 & 0 & 0 & 0 & 1 \\
\hline K. pneumoniae & 0 & 0 & 5 & 0 & 0 & 0 & 0 & 5 \\
\hline Proteus mirabilis & 0 & 0 & 0 & 4 & 0 & 0 & 0 & 4 \\
\hline S. aureus & 0 & 0 & 0 & 0 & 3 & 0 & 0 & 3 \\
\hline P. aeruginosa & 0 & 0 & 0 & 0 & 0 & 1 & & 1 \\
\hline K. oxytoca & 1 & 0 & 0 & 0 & 0 & 0 & 1 & 2 \\
\hline Total & 15 & 1 & 5 & 4 & 3 & 1 & 1 & 30 \\
\hline
\end{tabular}

Culture positivity of $50 \%$ in our study is less as compared to other studies $[33,34]$. Our setting being a tertiary care hospital in a country where antibiotics are available over the counter could be considered contributing to less culture positivity. The patients might have reported to other health care settings and received antimicrobials before presenting to our hospital.

4.2.1. Stone Matrices Culture and Infective Stone. Whole stone of 32 patients $(36.4 \%)$ yielded the growth of bacteria, whereas nidus had a positive result in $30(34.1 \%)$ of the cases. The common organisms isolated from whole stone and nidus were Escherichia coli followed by Klebsiella pneumoniae, P. mirabilis, E. faecalis, and S. aureus. Infective stone was found in $34.1 \%$. The urease producing and citrate utilizing organisms formed 14 (46. 7\%) and 12 (40\%), respectively. Whole stone culture was positive in 2 patients with the same organism isolated from urine culture and sterile nidus culture, indicating the stone formation as infection induced.

It has been hypothesized that alteration in urinary enzymes, i.e., decreased urokinase and increased sialidase in urine, leads to the formation of mineralizable matrix. $\mathrm{Mi}$ croorganisms like Proteus mirabilis and Escherichia coli associated with infection-induced stones inhibited the urokinase and stimulated the sialidase activity leading to matrix formation, in turn causing increased crystal adherence to the renal epithelium [35].

An alternative explanation for the presence of bacteria within stone and urine is that of secondary ascending infection from the bladder urine. Penetration of bacteria in the stone prevents complete eradication of urinary tract infection by conventional antibiotic therapy, allowing the development of resistant organisms with intermittent shedding in urine. It is a vicious cycle of infection bringing about stone formation and stone formation causing infection [36, 37].

4.2.2. Association of Bacteria Isolated from Urine and Stone Matrices Culture of the Patients. A total of 30 bacteria were isolated from stone formers and 28 had isolates in both urine and stone matrices, whereas in 2 patients, urine was sterile. Among 28 stone formers, in both urine and stone matrices, 24 had the same organism phenotypically and 3 had different strains of $E$. coli found in urine and stone matrices, while the remaining one had different isolates, i.e., E. coli from urine and K. oxytoca from stone matrices. Bacteria isolated from urine were common to stone matrices in context of whole stone culture also. The concordance rate between urine culture and stone culture varied from 16\% [38], 48 4\% [39], $50 \%$ [40], and $70 \%$ [4].

Our result also showed that the bacteria isolated from urine were associated significantly $(P$ value $<0.001)$ to nidus of stone which is considered gold standard. Though the concordance rate and sensitivity were high (93.3\%), the specificity and PPV were only $72.4 \%$ and $63.6 \%$, respectively. Antimicrobial susceptibility pattern of the bacteria isolated from infective stone was different than that from urinary isolates. Most of the microorganisms isolated from infective stone were multidrug resistant.

4.3. Stone Analysis. Stones from all 88 patients were subjected to qualitative biochemical analysis. The most common type of stone was calcium oxalate $(n=74,84.1 \%)$; others 


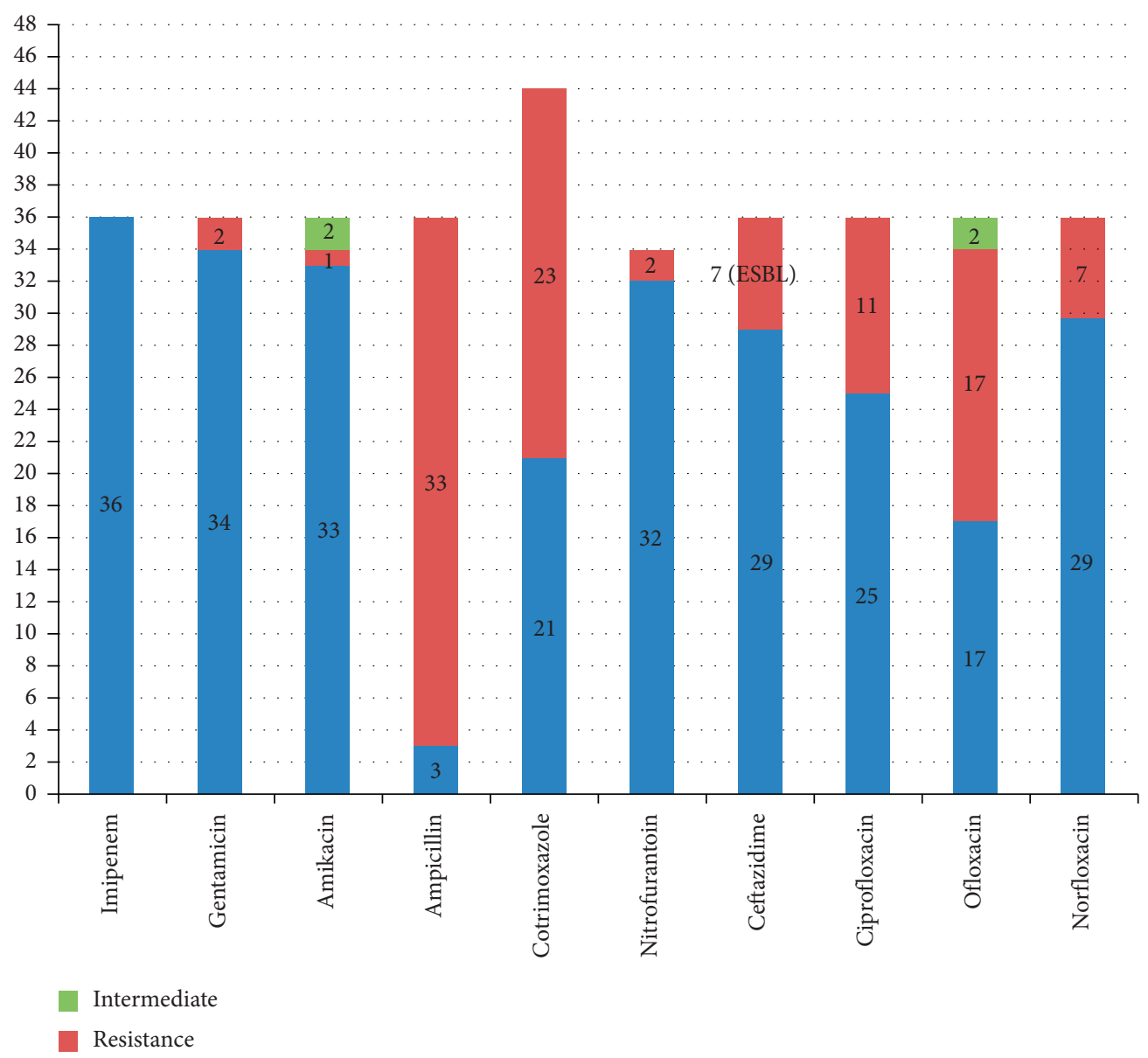

FIgURE 1: Antimicrobial susceptibility pattern of Gram-negative bacteria.

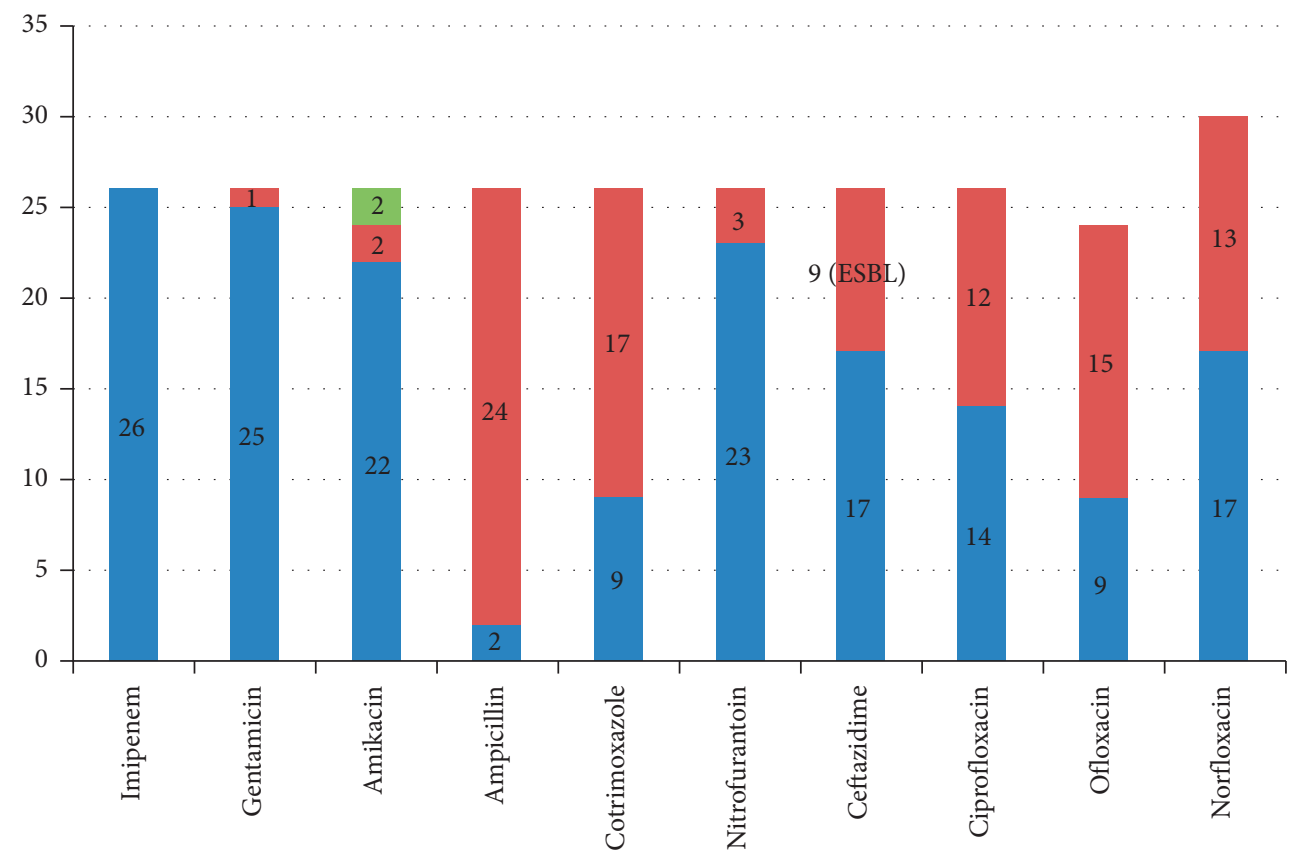

Intermediate

resistance

FIgURE 2: Nidus-antimicrobial susceptibility pattern of Gram-negative bacteria. 


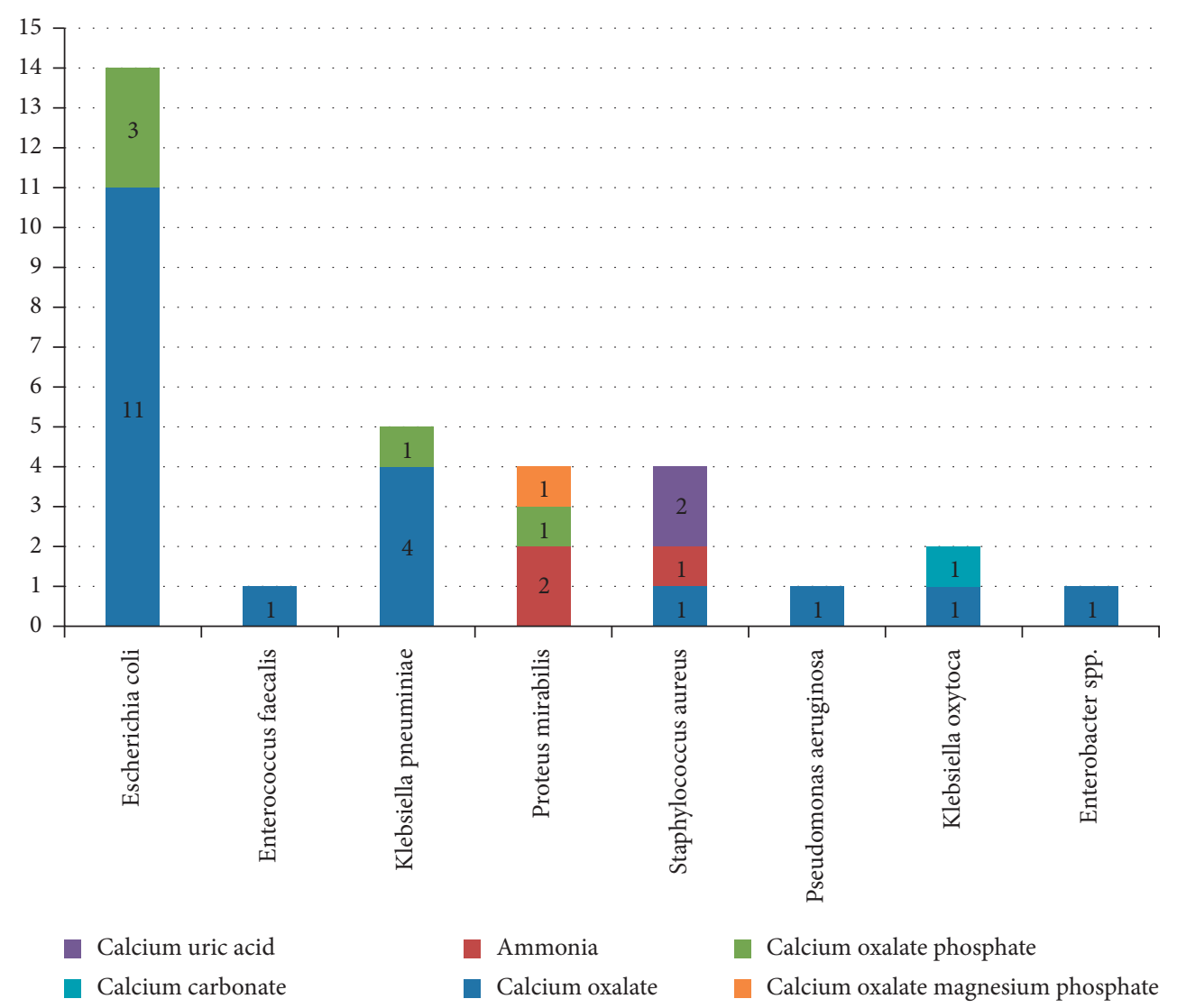

Figure 3: Stone bacteria with stone composition.

were calcium oxalate phosphate $(n=7,8.0 \%)$, ammonia $(n=3,3.4 \%)$, calcium uric acid $(n=2,2.3 \%)$, and calcium carbonate $(n=1,1.1 \%)$.

The infective stone accounted for 23 (71.9\%) out of the 74 calcium oxalate stones determined. The most common stone formers were $E$. coli followed by $K$. pneumoniae. Proteus mirabilis was associated with ammonia and calcium oxalate with magnesium and phosphate. S. aureus formed the calcium oxalate stone in mixed form with uric acid and ammonia. The bacterial isolates from nidus culture were associated with almost all types of urinary stones.

Several studies have found out the composition of staghorn stones which were considered to be a major portion of infective stones. Earlier studies demonstrated that calcium phosphate and struvite were major constituents of $75 \%$ of infective stones [41].

There was only one struvite stone in our study that too occurred in combination with calcium oxalate. This could be attributed to the smaller number of urease producing bacteria Proteus spp. In addition, other factors such as anatomic or functional abnormalities of urinary tract and diet may be responsible.

The present study supports studies from north India and Brazil, which reported calcium oxalate as the most common constituent of infective stones [42, 43].

As reported in literature, dietary habits are associated with urinary tract stones. Increased animal protein, high calorie content, as well as calcium and oxalate intake in the diet [44] are considered nutritional risk factors for urolithiasis. A study from Marathwada, India, has shown that the incidence of urolithiasis increases with consumption of diet containing groundnuts, tomato, spinach, and animal proteins and with a greater use of salt. Stone composition, urinary risk factors, and dietary analysis suggest that diet, dehydration, and poor nutrition are the causative factors of stone diseases [45].

Our study showed that calcium oxalate was the most common urinary tract stones, a finding consistent with that of Trinchieri [46] and Pandeya et al. [1]. One of the reasons for the occurrence of this type of stone has been cited as the consumption of nonvegetarian diet by the subjects as animal protein is found to lower the citrate excretion and increase calcium and uric acid excretion.

Diet with high oxalate content and high carbohydrate intake is also thought to be responsible for the occurrence of oxalate stones, particularly rice, and is found to increase the acidity of urine favoring calcium oxalate stone formation [1].

The results of the present study have indicated that infective stones constitute an accountable portion of urolithiasis with several types of microorganisms and with variable degrees of antimicrobial resistance. To understand the recurrent urinary infections, it is necessary to identify organism found deep in the stone matrices, which is not easily accessible to antimicrobial agents. Thus, this study has tried to aid information regarding the role of microbial agents as etiology of stone formation along with their 
antimicrobial susceptibility pattern so that appropriate antimicrobial therapy can be instituted not only for eradication of these agents but also for the prevention of recurrence.

\section{Conclusion}

The present study underscores the importance of microbiological analysis of stones for complete sterilization of urinary system and prevention of recurrence. In addition, this study recommends a further work on microbiological analysis of stones along with the correlation of various metabolic and dietary factors which may provide better insights regarding the origin of the stones in the urinary tract.

\section{Abbreviations}

AST: Antimicrobial susceptibilitytest

BHI: Brain-heart infusion broth

$\mathrm{CFU} / \mathrm{ml}$ : Colony-forming units per milliliter

CLSI: Clinical Laboratory Standards Institute

CLED: Cystein lactose electrolyte deficient

CONS: Coagulase negative staphylococci

ESBL: Extended spectrum beta lactamase

MHA: Muller-Hinton agar

MRSA: Methicillin-resistant Staphylococcus aureus

NPV: $\quad$ Negative predictive value

PPV: $\quad$ Positive predictive value

UTIs: Urinary tract infections

\section{Data Availability}

The data used to support the findings of this study are available from the corresponding author upon request.

\section{Ethical Approval}

Ethical approval has been taken from Institutional Review Committee (IRC code no.: IERB/277/014).

\section{Consent}

Informed consent was taken from the patients.

\section{Conflicts of Interest}

The authors declare that there are no conflicts of interest.

\section{Authors' Contributions}

PS conceptualized and designed the study, obtained data acquisition, and prepared the manuscript. RB and $\mathrm{ML}$ performed literature search and analysed data. CSA prepared and edited the manuscript. DDB performed statistical analysis. BK prepared, edited, and reviewed the manuscript.

\section{References}

[1] D. R. Pandeya, D. Adhikari, S. Risal, J. Baxi, and P. P. Singh, "Epidemiology and etiopathogenesis of urinary calculi in western Nepal," Nepal Medical College Journal, vol. 8, no. 3, pp. 190-193, 2006.

[2] R. Tavichakorntrakoo, V. Prasongwattana, S. Sungkeeree et al., "Extensive characterizations of bacteria isolated from catheterized urine and stone matrices in patients with nephrolithiasis," Nephrology Dialysis Transplantation, vol. 27, no. 11, pp. 4125-4130, 2012.

[3] K.-H. Bichler, E. Eipper, K. Naber, V. Braun, R. Zimmermann, and S. Lahme, "Urinary infection stones," International Journal of Antimicrobial Agents, vol. 19, no. 6, pp. 488-498, 2002.

[4] H. Shafi, Z. Shahandeh, B. Heidari et al., "Bacteriological study and structural composition of staghorn stones removed by the anatrophic nephrolithotomic procedure," Saudi Journal of Kidney Diseases and Transplantation, vol. 24, no. 2, pp. 418-423, 2013.

[5] D. P. Griffith, J. R. Gibson, C. W. Clinton, and D. M. Musher, "Acetohydroxamic acid: Clinical studies of a urease inhibitor in patients with staghorn renal calculi," Journal of Urology, vol. 119, no. 1, pp. 9-15, 1978.

[6] D. P. Griffith, P. A. Moskowitz, and C. E. Carlton Jr, "Adjunctive chemotherapy of infection-induced staghorn calculi," Journal of Urology, vol. 121, no. 6, pp. 711-715, 1979.

[7] J. Sobel and D. Kaye, "Urinary tract infection,"'Urinary tract infection," in Mandell, Douglas and Bennett's Principles and Practice of Infectious Disease, G. L. Mandell, J. E. Benett, and R. Dolin, Eds., pp. 957-983, Elsevier, Amsterdam, Netherlands, 7th edition, 2010.

[8] Mackie \& McCartney, Practical Medical Microbiology, pp. 84-88, Churchill Livingstone, London, UK, 14th edition, 2011.

[9] W. Winn, S. Allen, W. Janda, E. Koneman et al., Koneman's Color Atlas and Textbook of Diagnostic Microbiology, Jones \& Bartlett Learning, Burlington, MA, USA, 6th edition, 2006.

[10] Clinical and Laboratory Standard Institute, Performance Standard for Antimicrobial Disk Susceptibility Tests, Document No M100-S23, Clinical and Laboratory Standard Institute, Wayne, PA, USA, 23rd edition, 2013.

[11] A. H. Gowenlock, Varley's Practical Clinical Biochemistry, Heinemann Medical Books, London, UK, 6th edition, 1996.

[12] J. C. Lieske, L. S. Peña de la Vega, J. M. Slezak et al., "Renal stone epidemiology in Rochester, Minnesota: An update," Kidney International, vol. 69, no. 4, pp. 760-764, 2006.

[13] S. Nowfar, K. Palazzi-Churas, D. C. Chang, and R. L. Sur, “The relationship of obesity and gender prevalence changes in United States inpatient nephrolithiasis," Urology, vol. 78, no. 5, pp. 1029-1033, 2011.

[14] J. Fan, P. S. Chandhoke, and S. A. Grampsas, "Role of sex hormones in experimental calcium oxalate nephrolithiasis," Journal of the American Society of Nephrology, vol. 10, no. Suppl 14, pp. S376-S380, 1999.

[15] H. J. Heller, K. Sakhaee, O. W. Moe, and C. Y. C. Pak, "Etiological role of estrogen status in renal stone formation," Journal of Urology, vol. 168, no. 5, pp. 1923-1927, 2002.

[16] M. R. Naghii, M. Babaei, and M. Hedayati, "Androgens involvement in the pathogenesis of renal stones formation," PLoS One, vol. 9, no. 4, Article ID e93790, 2014.

[17] K. Gupta, G. Gill, and R. Mahajan, "Possible role of elevated serum testosterone in pathogenesis of renal stone formation," International Journal of Applied and Basic Medical Research, vol. 6, no. 4, pp. 241-244, 2016.

[18] E. W. Vahlensieck, D. Bach, and A. Hesse, "Incidence, prevalence and mortality of urolithiasis in the German Federal 
Republic," Urological Research, vol. 10, no. 4, pp. 161-164, 1982.

[19] M. A. Kadir, M. N. Ibrahim, and N. M. Salih, "Prevalence of urinary tract infections in patients with renal stones,".

[20] S. C. Kim, Y. T. Moon, Y. P. Hong et al., "Prevalence and risk factors of urinary stones in Koreans," Journal of Korean Medical Science, vol. 13, no. 2, pp. 138-146, 1998.

[21] A. Losito, E. G. Nunzi, C. Covarelli, E. Nunzi, and G. Ferrara, "Increased acid excretion in kidney stone formers with essential hypertension," Nephrology, Dialysis, Transplantation, vol. 24, no. 1, pp. 137-144, 2009.

[22] Y. Kohjimoto, Y. Sasaki, M. Iguchi, N. Matsumura, T. Inagaki, and I. Hara, "Association of metabolic syndrome traits and severity of kidney stones: Results from a nationwide survey on urolithiasis in Japan," American Journal of Kidney Diseases, vol. 61, no. 6, pp. 923-929, 2013.

[23] L. Wang, C. Feng, G. Ding et al., "Correlative anaylsis between clinical patterns of urolithiasis and laboratory parameters and evaluation of risk factors in calculous kidney damage," International Journal of Clinical and Experimental Medicine, vol. 9, no. 9, pp. 18419-18426, 2016.

[24] B. H. Eisner, S. P. Porten, S. K. Bechis, and M. L. Stoller, "Hypertension is associated with increased urinary calcium excretion in patients with nephrolithiasis," Journal of Urology, vol. 183 , no. 2 , pp. $576-579,2010$.

[25] N. Meydan, S. Barutca, S. Caliskan, and T. Camsari, "Urinary stone disease in diabetes mellitus," Scandinavian Journal of Urology and Nephrology, vol. 37, no. 1, pp. 64-70, 2003.

[26] R. Nerli, M. Jali, A. K. Guntaka, P. Patne, S. Patil, and M. B. Hiremath, "Type 2 diabetes mellitus and renal stones," Advanced Biomedical Research, vol. 4, p. 180, 2015.

[27] F.-Y. Chu, C.-C. Chang, P.-H. Huang et al., "The association of uric acid calculi with obesity, prediabetes, type 2 diabetes mellitus, and hypertension," BioMed Research International, vol. 2017, pp. 1-7, Article ID 7523960, 2017.

[28] M. Daudon, O. Traxer, P. Conort, B. Lacour, and P. Jungers, "Type 2 diabetes increases the risk for uric acid stones," Journal of the American Society of Nephrology, vol. 17, no. 7, pp. 2026-2033, 2006.

[29] F. L. Coe, J. H. Parks, and J. R. Asplin, "The pathogenesis and treatment of kidney stones," New England Journal of Medicine, vol. 327, no. 16, pp. 1141-1152, 1992.

[30] J. Alvarez-Nemegyei, M. Medina-Escobedo, S. VillanuevaJorge, and J. Vazquez-Mellado, "Prevalence and risk factors for urolithiasis in primary gout: is a reappraisal needed?" The Journal of Rheumatology, vol. 32, pp. 2189-2191, 2005.

[31] I. Ahmad, M. S. Pansota, M. Tariq, and S. A. Tabassum, "Frequency of metabolic abnormalities in urinary stones patients," Pakistan Journal of Medical Sciences, vol. 29, no. 6, pp. 1363-1366, 2013.

[32] M. Venkatramana, J. Muttappallymyalil, J. Sreedharan et al., "Age at onset and clinical presentation of urolithiasis in Ajman, UAE," Australasian Medical Journal, vol. 3, no. 10, pp. 662-666, 2010.

[33] T. Naas, S. Al-Agili, and O. Bashir, "Urinary calculi: Bacteriological and chemical association," Eastern Mediterranean Health Journal, vol. 7, no. 4-5, pp. 763-770, 2001.

[34] H. Jan, I. Akbar, H. Kamran et al., "Frequency of renal stone disease in patients with urinary tract infection," Journal of Ayub Medical College Abbottabad, vol. 20, no. 1, pp. 60-62, 2008.

[35] P. J. du Toit, C. H. van Aswegen, P. L. Steyn, A. Pols, and D. J. du Plessis, "Effects of bacteria involved with the pathogenesis of infection-induced urolithiasis on the urokinase and sialidase (neuraminidase) activity," Urological Research, vol. 20, no. 6, pp. 393-397, 1992.

[36] N. J. Nemoy and T. A. Stainey, "Surgical, bacteriological, and biochemical management of infection stones," JAMA: The Journal of the American Medical Association, vol. 215, no. 9, pp. 1470-1476, 1971.

[37] J. E. A. Wickham, "Matrix and the Infective renal calculus," British Journal of Urology, vol. 47, no. 7, pp. 727-732, 1976.

[38] R. Devraj, K. Tanneru, B. Reddy, H. Amancherla, and R. Chilumala, "Renal stone culture and sensitivity is a better predictor of potential urosepsis than pelvic or midstream urine culture and sensitivity," Journal of Dr. NTR University of Health Sciences, vol. 5, no. 4, pp. 261-264, 2016.

[39] S. Golechha and A. Solanki, "Bacteriology and chemical composition of renal calculi accompanying urinary tract infection," Indian Journal of Urology, vol. 17, pp. 111-117, 2001.

[40] M. R. de Cógáin, J. C. Lieske, T. J. Vrtiska, P. K. Tosh, and A. E. Krambeck, "Secondarily infected nonstruvite urolithiasis: A prospective evaluation," Urology, vol. 84, no. 6, pp. 1295-1300, 2014.

[41] I. Rosenstein, R. S. Osborn, J. P. Hopewell, J. M. HamiltonMiller, and W. Brumfitt, "Bacteriological and crystallographical analysis of urinary calculi: Aid to patient management," Journal of the Royal Society of Medicine, vol. 77, no. 6, pp. 478-482, 1984.

[42] M. S. Ansari, N. P. Gupta, A. K. Hemal et al., "Spectrum of stone composition: structural analysis of 1050 upper urinary tract calculi from northern India," International Journal of Urology, vol. 12, no. 1, pp. 12-16, 2005.

[43] S. F. Ribeiro da Silva, S. Leite da Silva, E. De Francesco Daher, H. de Holanda Campos, and C. A. Bruno da Silva, "Composition of kidney stone fragments obtained after extracorporeal shock wave lithotripsy," Clinical Chemistry and Laboratory Medicine, vol. 48, no. 3, pp. 403-404, 2010.

[44] R. Siener, "Impact of dietary habits on stone incidence," Urological Research, vol. 34, no. 2, pp. 131-133, 2006.

[45] L. Borghi, T. Meschi, F. Amato, A. Briganti, A. Novarini, and A. Giannini, "Urinary volume, water and recurrences in idiopathic calcium nephrolithiasis," The Journal of Urology, vol. 155 , pp. 839-843, 1996.

[46] A. Trinchieri, "Epidemiology of urolithiasis: An update," Clinical Cases in Bone and Mineral Metabolism, vol. 5, no. 2, pp. 101-106, 2008. 